\title{
Dynamical modeling and the interactions with the ISM
}

\author{
Wolfgang Steffen $\dagger$ \\ Instituto de Astronomía, Universidad Nacional Autónoma de México, Ensenada, Mexico \\ email: wsteffen@astrosen.unam.mx
}

\begin{abstract}
This paper is a review of some of the recent modeling efforts to improve our understanding of structure formation and evolution of planetary nebulae including their interaction with the interstellar medium. New propositions have been made for the formation mechanism of multi-polar PNe and PPNe. These mechanisms are based on the central engine with interacting binary stars or hole producing instabilities in expanding shock waves leading to illumination effects from the central star that change the appearance of the nebula. Furthermore, there has been a lot of progress in the observation and 3D modeling of the kinematics, which is key to the understanding of the dynamics. Extensive observational catalogs are coming online for the kinematics, as well as some very detailed proper motion measurements have been made. New techniques for morpho-kinematic 3D modeling help to make the interpretation of kinematic data more reliable and detailed. In addition to individual pointed observations, new surveys have lead to the discovery of many PNe that show clear signs of interaction with the interstellar medium. Systematic hydrodynamic models of the interaction have produced a general scheme for the observed structure that results from the interaction of an evolving planetary nebula with the ISM. Detailed modeling of the dust-gas dynamics during the interaction with the ISM have produced interesting predictions for future IR observations. Detailed models were worked out for the structure of the bowshock and tail of Mira that was recently discovered in the UV.
\end{abstract}

Keywords. Planetary nebulae: general, hydrodynamics, ISM: kinematics and dynamics, ISM: magnetic fields

\section{Introduction}

In general terms the evolution of structure in planetary nebulae $(\mathrm{PNe})$ appeared to have been pretty much solved with the interacting winds model by Kwok et al.(1978) and its generalization (Kahn \& West, 1985; Balick 1987). A lot of theoretical work with numerical simulations during the nineties was able to reproduce findings from imaging and observations of the kinematics from spectroscopy (e.g. Mellema et al., 1991; GarcíaSegura \& López, 2000). From this illuminating research it seemed reasonable that the time scale for the structure formation was of the order of a thousand years or so. Hubble Space Telescope (HST) observations of proto-planetary nebulae (PPNe) then made it clear that the complex structure must have been formed at least an order of magnitude earlier and at a smaller spatial scale (Aaquist \& Kwok, 1996). Therefore research trend is now going towards zooming in on nature of the "central engine", i.e. the central star(s) and the immediate surrounding on the scale of the size of an AGB star.

In this paper I shall not consider the work that has been done on the central engine itself, but look at the research on the structure further out that might help to constrain

$\dagger$ Present address: Institut für Computergraphik, Technische Universität Braunschweig, Mühlenpfordstr. 23, 38118 Braunschweig, Germany 

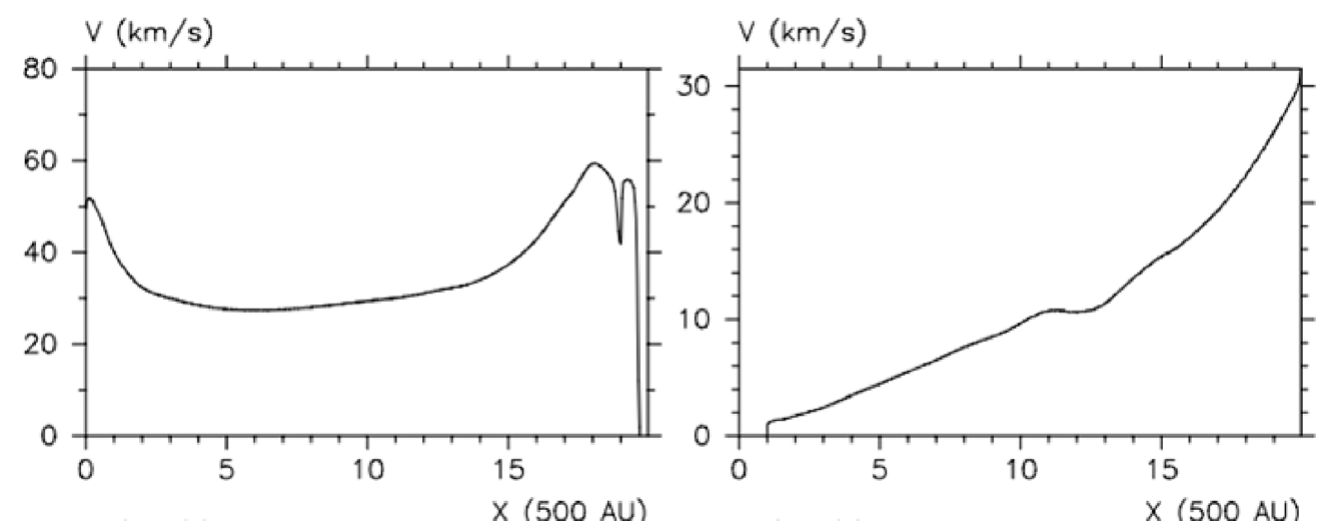

Figure 1. The weighted average velocity field produced by a jet (right) and a bullet as found by Dennis et al. (2008).

the nature of the inner workings at scales that are spatially resolved with current imaging techniques. A key constraint for the dynamics, in addition to the morphology, is of course the kinematics. Therefore, some emphasis will be put on the research that has been done in modeling observed kinematics with hydrodynamic simulations as well as morpho-kinematic modeling, including new techniques that have been developed for the interpretation of observational data.

\section{Jets and bullets}

Jets and bullets have been suggested as the origin of the collimated features that can be observed in a number of young PNe and PPNe, such as He2-115 (Sahai \& Trauger, 1998) or CRL 618 (Trammel \& Goodrich, 2002). Raga et al. (2007) investigated the disintegration of bullets with high resolution 3-D hydrodynamical simulations and find that the debris from the bullet assumes a velocity distribution that is roughly linear with distance to a reference position. Dennis et al. (2008) looked at the shape and the velocity field of the bowshock or elongated lobes that bullets and jets inflate during their propagation through the local environment. They find that the lobes from continuous jets are "thicker", i.e. have a lower ratio between length and diameter, as compared to the bullets. Furthermore, the velocity profile of the lobes is rather flat whereas that of the bullets shows a continuous nearly linear increase of velocity with distance from the source (see Fig. 1). Their results suggest that it should be possible to distinguish between an origin from bullets and jets using detailed observations of the kinematics of the lobes.

Further research into the effects of knots and bullets on spectral line shapes and ratios was done by Raga et al. (2008). They find that from the observed spectral line ratios it is hard or impossible to distinguish whether the structure is a stationary or slow object that is mainly photo-ionized or is a shock-ionized fast region. It is therefore necessary to include kinematic data to distinguish between rather stationary photo-ionized structure and high-velocity shocked structures. Velázquez et al. (2007) performed hydrodynamical simulations to model the properties of the water-maser source K3-35, finding that they were able to reproduce the radio morphology and kinematics with a precessing jet that has a period of 100 years and a semi-opening angle of 20 degrees. Guerrero et al. (2008) performed a detailed observational analysis of IC 4634 and included hydrodynamical simulations of precessing variable jets to test their interpretation of the observations. 

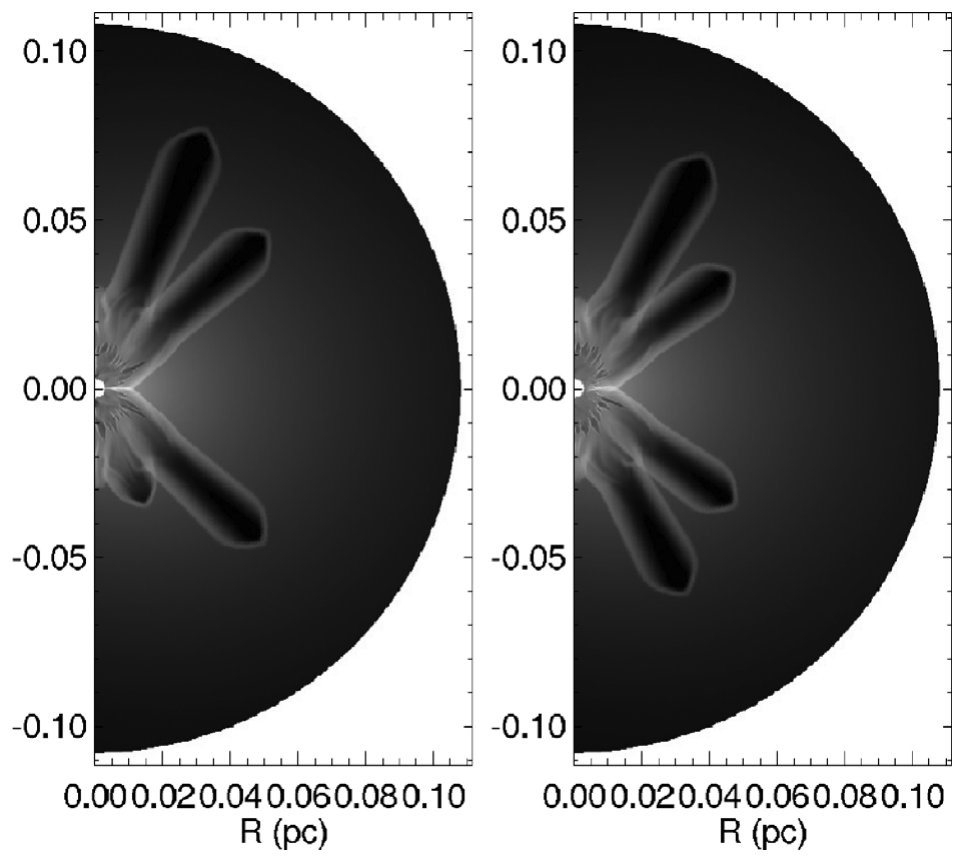

Figure 2. Cuts through the density distribution of hydrodynamical simulations of the multi-polar structure formations through instabilities in photo-dissociation regions is shown (García-Segura 2011)

In the past it has been rare to see hydrodynamical simulations that were tailored for a particular object. With progress in numerical computing capabilities, this is more viable now. Other examples for such simulations are those presented by Monteiro \& FalçetaGonçalves (2011; see also their poster in these proceedings) for NGC 40. They used to their dynamical simulations as input to a detailed photoionization model. Velázquez et al. (2011) performed simulations for the Red Rectangle.

Akashi \& Soker (2008) did a detailed study on shaping PNe with light jets and partially collimated winds. In Akashi et al. (2008) they compared the X-ray emission properties in PNe that have been inflated by jets and partially collimated winds to investigate the problem of low observed X-ray temperatures. They find the hot shocked gas could enough through adiabatic expansion to explain the observations. However, for less collimated fast winds require heat conduction to explain the low temperatures of the X-ray emitting gas. This is agrees with earlier results from simulations of spherical winds by Steffen et al. (2008).

\section{Multi-polar lobes}

One of the most surprising discoveries about PPNe and PNe with HST-class highresolution observations was the finding of clearly multipolar objects such as CRL 618 . The traditional interacting wind models are unable to explain this structure. The first thought was then to introduce highly collimated ejections, like jets or bullets, that vary in direction and intensity over time (Sahai \& Trauger 1998; Sahai 2000). The time scale of this variability must be rather small, of the order or tens of years or so (see e.g. Riera et al., these proceedings). Recently, Kwok (2010) proposed that bipolar and multipolar 


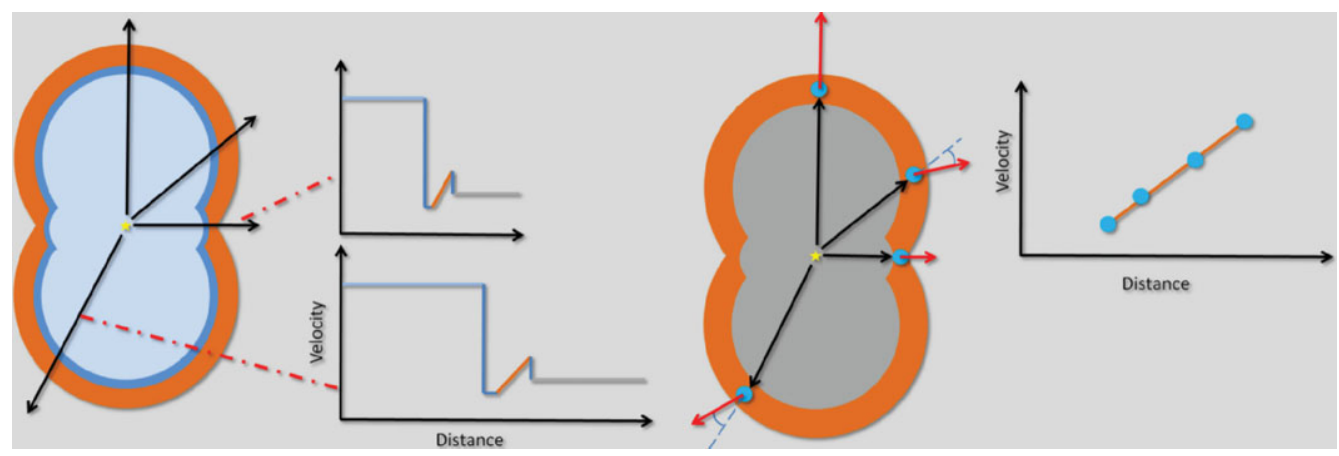

Figure 3. The radial velocity field in a PN is usually rather complex and is linear only over a small range (left). Although the shape of the velocity field may be similar in different directions, quantitatively it will be different. When considering a single "shell" (right), the plot of the speed versus distance will be closer to a linear increase, although usually it still differs from a homologous expansion law, especially because of deviations from the radial direction.

lobes of PNe could result from illumination and ionization effects alone, without the need of collimated ejections. Based on this hypothesis, García-Segura (2011) developed a multipolar structure forming model that is based on photo-dissociation before the central star is hot enough to ionize its environment. This model explains multipolar features to appear during the PPN-phase and that may persist well into the evolved PN-phase (Fig. 2).

\section{Velocity and metallicity}

The metallicity of ionized hot gas has a strong impact on its radiation and therefore also on its cooling properties. As a result, also the dynamical evolution will be influenced by the metallicity. Schönberner et al. (2010) have used spherically symmetric radiationhydrodynamic simulations to investigate the impact of metallicity on the expansion of PNe. They find that the metallicity strongly changes the radial structure and velocity field. "The lower the metal content, the larger and thicker become the nebular shells and the smaller the wind-blown cavities", which is, of course, similar to the difference between adiabatic (no radiative losses) and isothermal expansion (dynamical heating is off-set by strong radiative losses). Their paper is very extensive and provides a detailed quantitative analysis of the influence of metallicity on the structure and kinematics.

\section{Morpho-kinematic modeling}

Since the advent of the HST, the observed complexity of PNe has increased dramatically and with it the potential of miss-classification due to orientation effects. Considerable effort has therefore been invested to improve the reconstruction of the 3-D structure from imaging and kinematic data (e.g. Sabbadin et al. 2004; Steffen \& López 2006; Steffen et al. 2011; Hajian et al. 2007). Usually, a key ingredient to these reconstructions is the assumption of a homologous expansion of the nebula, i.e. the velocity vector is proportional to position. Such a velocity field provides a linear mapping between the velocity (that can be measured with spatially resolved spectroscopy) and the position along the line of sight. Assuming the existence of some symmetry - such as sphericity or a cylindrical symmetry - the 3-D structure can be reconstructed (including the inclination angle). This type of expansion is expected from a ballistic explosion. However, the 
(a)

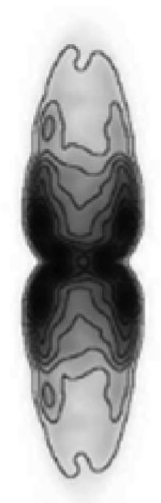

(b)

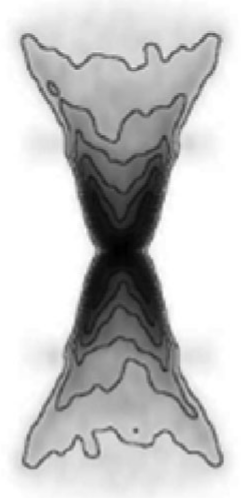

(c)

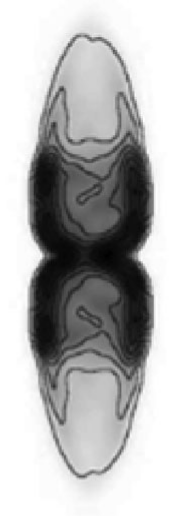

$90^{\circ}$
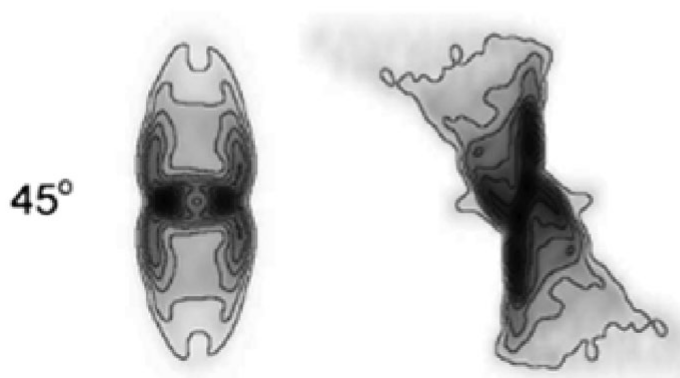

Figure 4. A hydrodynamic simulation of a cylindrically symmetric bipolar PN is show at two different viewing angles (col. a). Col. (b) is a reconstruction assuming a homologous expansion and as seen from a direction in the plane of the sky. In col. (c) the same view is shown but as reconstructed with the correct velocity law. The figure is from Steffen et al. (2009).

generalized interacting wind (GIW) scenario implies a non-spherical continuously driven flow for a significant time of the nebular evolution, such that we can not expect a ballistic flow. If we find that the velocity field is homologous with a precision that is inconsistent with the GIW model, then the driving force for the structure formation must have acted only for a very short period compared to the current age of a nebula.

Steffen et al. (2009) showed how the non-homologous velocity field may affect the reconstruction of structure along the line of sight (Fig. 4) if a homologous expansion is assumed. They used hydrodynamical simulation as a know "laboratory" object for the reconstruction. The result depends on the inclination angle and can produce misclassifications. Fig. (4) shows the same simulated object (col. a) and its reconstruction (col. b) for two different viewing angles. At 45 degrees the object might be falsely classified as point-symmetric. Column (c) shows the reconstruction when the correct velocity field is taken into account. The problem is, that either the velocity field has to be assumed or a strong symmetry (like cylindrical symmetry) in order to have a unique reconstruction. Sometimes symmetric properties of parts of an object may help to solve ambiguities. The recent development of the interactive morpho-kinematic software SHAPE has contributed to a considerable increase of very detailed 3-D reconstructions of a variety of PNe (Steffen et al., 2011, see also http://www.astrosen.unam.mx/shape).

A key new kind of information to improve 3-D models of the structure and velocity field is beginning to appear from direct expansion and internal proper motion measurements. 


\section{direction of motion}
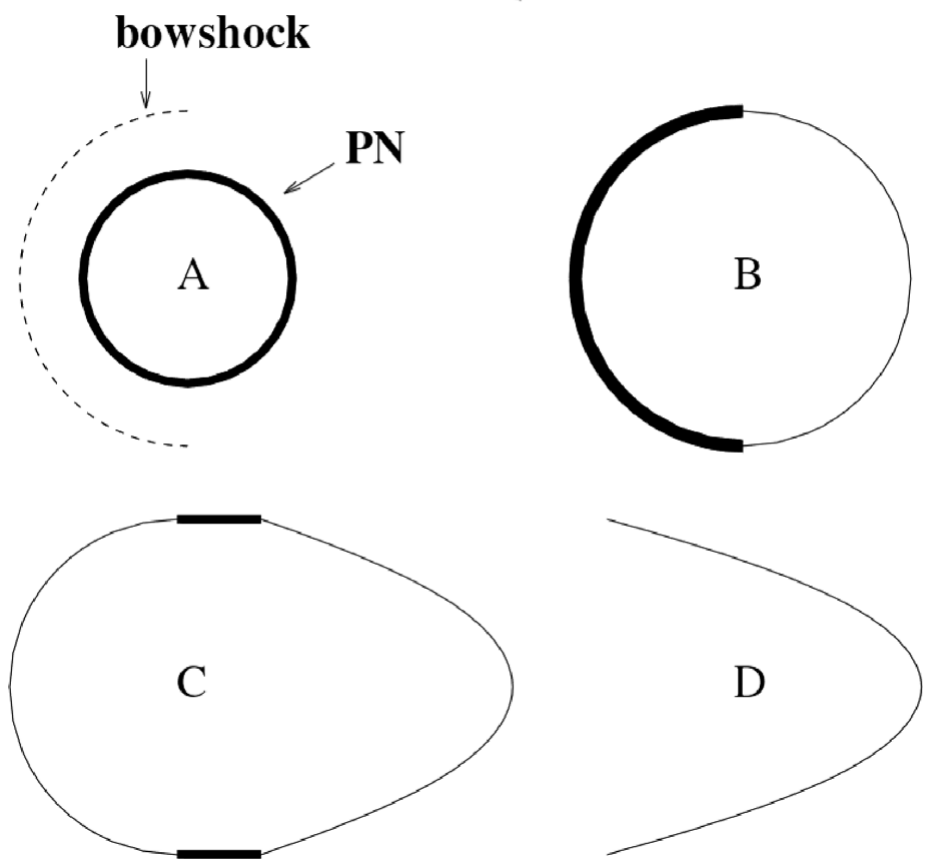

Figure 5. The classification scheme introduced by Wareing et al. (2007b) to describe the basic evolutionary stages of the interaction of PNe with the ISM. Small grains are on the left and large ones on the right.

The time baseline of multi-epoch HST observations is now large enough to measure the expansion component in the plane of the sky for the nearby objects ( $\mathrm{Li}$ et al. 2002; Szyszka et al. 2011). They complement the spectroscopic Doppler-velocities that are already available (see e.g. López et al., these proceedings). The emerging proper motion measurements promise to produce a whole new game in morpho-kinematic modeling. New analysis techniques specific to proper motion measurements are already being developed in the form of criss-cross mapping and distance mapping (e.g. Steffen \& Koning 2011; Akras \& Steffen, these proceedings). Radiation-hydrodynamic modeling to assess the impact of potential caveats for the interpretation of such data are needed, since there may be differences between the material flow as measured by the Doppler-effect and the pattern expansion near ionization fronts (Mellema 2004). Similarly, possible sources of systematic errors in the proper motion measurements should be evaluated, such as the overlapping of features from the front and back sides of a nebula.

\section{ISM interaction}

The increased number of discoveries of low-surface brightness evolved PNe in surveys like IPHAS (Sabin et al., 2010) and MASH (Parker et al. 2006) has revealed a number of objects that show signs of interaction with the interstellar medium (ISM). Asymmetries seen in these objects have to be distinguished from intrinsic structure that has its origin in the formation process of the nebula on a small scale. After the initial work by Villaver et al. (2003, 2005), Wareing et al. (2007b) have conducted a series of simulations that systematically explores the main structural patterns in PNe that interact with the ISM. 


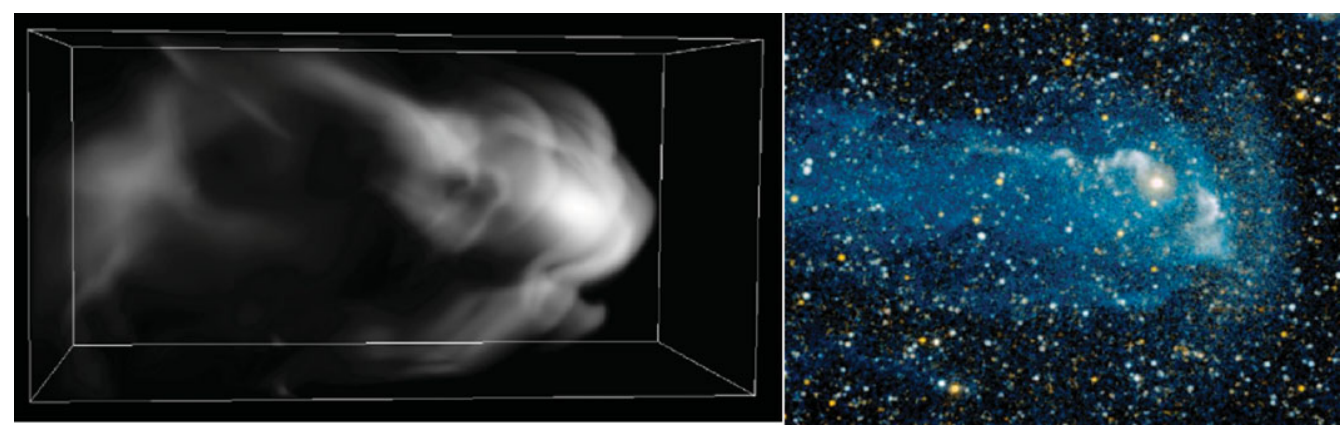

Figure 6. The bowshock around Mira has a very complex structure (right, GALEX, Martin et al. 2007). It was simulated by Raga et al. (2008) with a wind that has a latitude-dependent density structure and an axis that is oblique to the relative motion between the star and the ISM.

This has lead to a simple classification scheme that is based on the main, qualitatively different, phases of the interaction (Fig. 5).

Ueta et al. (2009) have devised a new method that uses the stellar wind interaction with the ISM to derive the 3-D ISM motion in the region near the star. They fit an analytical bowshock shape to the imaging observations in the IR and, with a few assumptions, obtain the velocity vector of the ISM in the star's neighbourhood.

The spectacular tail of Mira that was detected in the UV (Martin et al. 2007) has also been the subject of analytical calculations and numerical simulations. Wareing et al. (2007a) came to the conclusion that the tail was approx. 470 thousand years old and that the rebrightening of the tail at some distance from the star was due to the star entering the Local Bubble (LB). Raga et al. explored the details of the bowshock structure (Fig. 6) and the rebrightening with high resolution 2-D and 3-D simulations (Raga \& Cantó 2008; Raga et al. 2008). They also investigated the effects of Mira entering the LB in Esquivel et al. (2010) and confirmed that the rebrightening of Mira's tail could be due to penetrating the LB.

Ransom et al. (2008) consider that the presence and deformation of the ISM magnetic field could explain the polarization of radio emission observed in the PN Sh-2 216. They propose that the motion of the PN through the ISM compresses and deforms the otherwise more uniform field. This results in enhanced and polarized radio emission towards the head of the bowshock that can also be observed in the optical.

The dynamical motion of dust in a bowshock of an AGB wind was explored in a series of simulations by van Marle et al. (2011, see also their poster contribution in these proceedings). They find that the small grains conform to the density distribution of the gas whereas the larger grains follow their inertia and can not be held back as easily by the gas. They predict that there should be an imprint of the separation of grain sizes in the IR radiation. This phenomenon would be the largest known selective dust filter in the universe.

\section{Acknowledgements}

The author acknowledges support by Universidad Nacional Autónoma de México through grant DGAPA PAPIIT IN100401 and the Alexander von Humboldt Stiftung, as well as the support and the hospitality of the Institut für Computergraphik at the Technische Universität Braunschweig during his sabbatical year. 


\section{References}

Aaquist, O. B. \& Kwok, S. 1996, ApJ, 462, 813

Akashi, M. \& Soker, N. 2008, MNRAS, 391, 1063

Akashi, M., Meiron, Y., \& Soker, N. 2008, New Astronomy, 13, 563

Balick, B. 1987, AJ, 94, 671

Dennis, T. J., Cunningham, A. J., Frank, A., Balick, B., Blackman, E. G., \& Mitran, S. 2008, ApJ, 679, 1327

Esquivel, A., Raga, A. C., Cantó, J., et al. 2010, ApJ, 725, 1466

García-Segura, G. \& López, J. A. 2000, ApJ, 544, 336

García-Segura, G. 2011, A\& $A, 520$, L5

Guerrero, M. A., Miranda, L. F., Riera, A., Velázquez, P. F., et al. 2008, ApJ, 683, 272

Hajian, A. R., Movit, S. M., Trofimov, D., Balick, B., et al. 2007, ApJ, 169, 289

Kahn, F. D. \& West, K. A. 1985, MNRAS, 212, 837

Kwok, S., Purton, C. R., \& FitzGerald, P. M. 1978, ApJ, 219, L125-L127

Kwok, S. 2010, PASA, 27, 174

Li, J., Harrington, J. P., \& Borkowski, K., 2002, AJ, 123, 2676

Martin, D. C., Seibert, M., Neill, J. D., et al. 2007, Nature, 448, 780

Mellema, G., Euldernick, F., \& Icke, V. 1991, A\&A, 252, 718

Mellema, G. 2004, A\& A, 416, 623

Monteiro, H. \& Falçeta-Gonçalves, D. 2011, ApJ, 738, 174

Parker, Q. A., Acker, A., Frew, D. J., et al. 2006, MNRAS, 373, 79

Raga, A. C., Cantó 2008, ApJ, 685, 141

Raga, A. C., Cantó, de Colle, J., et al. 2008, ApJ, 680, 45

Raga, A. C., Esquivel, A., Riera, A., \& Velázquez, P. F. 2007, ApJ, 668, 1141

Raga, A. C., Riera, A., Mellema, G., Esquivel, A., \& Velázquez, P. F. 2008, A\&JJ, 489, 1141

Ransom, R. R., Uyaniker, B., Kothes, R., \& Landecker, T. L. 2008, A\&UJ, 684, 1009

Sabbadin, F., Turatto, M., Cappellaro, E., Benetti, S., \& Ragazzoni, R. 2004, A\&A, 416, 955

Sabin, L., Zijlstra, A. A., Wareing, C., et al. 2010, PASA, 27, 166

Sahai, R. 2000, ApJ, 537, L43

Sahai, R. \& Trauger, J. T. 1998, AJ, 116, 1357

Schönberner, D., Jacob, R., Sandin, C., \& Steffen, M. 2010, A\&A, 523, A86

Steffen, M., Schönberner, D., \& Warmuth, A. 2008, A\&A, 489, 173

Steffen, W. \& López, J. A. 2006, RevMexAA, 42, 99

Steffen, W. \& Koning, N., 2011, AJ, 141, A76

Steffen, W., Koning, N., Wenger, S., Morisset, C., \& Magnor, M., 2011, IEEE Transactions on Visualization and Computer Graphics, vol. 17 no. 4, pp. 454

Steffen, W., García-Segura, G., \& Koning, N., 2009, ApJ, 691, 696

Szyszka, C., Zijlstra, A. A., \& Walsh, J. R., 2011, MNRAS, 416, 715

Trammell, S. R. \& Goodrich, R. W. 2002, ApJ, 579, 688

Ueta, T., Uzumiura, H., Yamamura, I., et al. 2009, AKARI, a Light to Illuminate the Misty Universe, ASP Conf. S., 418, 117

van Marle, A. J., Meliani, Z., Keppens, R., \& Decin, L. 2011, ApJ, 734, 26

Velázquez, P. F., Gómez, Y., Esquivel, A., \& Raga, A. C. 2007, MNRAS, 382, 1965

Velázquez, P. F., Steffen, W., Raga, A. C., Haro-Corzo, S., Esquivel, A., Cantó, J., \& Riera, A. 2011, ApJ, 734, 57

Villaver, E., García-Segura, G., \& Manchado, A. 2003, ApJ, 585, L49

Villaver, E. \& Stanghellini, L. 2005, ApJ, 632, 854

Wareing, C. J., Zijlstra, A. A., O'Brien, T. J., \& Seibert, M. 2007a, ApJ, 670, 125

Wareing, C. J., Zijlstra, A. A., \& O'Brien, T. J. 2007b, MNRAS, 382, 1233 